\title{
High Sensitivity Refractive Index Sensor Based on Splicing Points Tapered SMF-PCF-SMF Structure Mach-Zehnder Mode Interferometer
}

\author{
Qi Wang ${ }^{1,2, *}$, Lingxin Kong ${ }^{1}$, Yunli Dang ${ }^{1}$, Feng Xia ${ }^{1}$, Yongwei Zhang ${ }^{1}$, Yong Zhao ${ }^{1,2}$, Haifeng \\ $\mathrm{Hu}^{1}$, and ${\mathrm{Jin} \mathrm{Li}^{1}}^{1}$ \\ ${ }^{1}$ College of Information Science and Engineering, Northeastern University, Shenyang 110819, China
}

${ }^{2}$ State Key Laboratory of Synthetical Automation for Process Industries (Northeastern University), Shenyang 110819, China

*Corresponding author Email: wangqi@ise.neu.edu.cn

\section{Corresponding Author: Qi Wang}

Qi Wang is with College of Information Science and Engineering, Northeastern University, and State Key Laboratory of Synthetical Automation for Process Industries (Northeastern University).

Email: wangqi@ise.neu.edu.cn

Telephone number: $+86-13889195280$

Mailing address:

Northeastern University, College of Information Science and Engineering

P. O. Box 321, Shenyang, Liaoning, 110819, China

Lingxin Kong, Yunli Dang, Feng Xia, Yongwei Zhang, Yong Zhao, Haifeng Hu and Jin Li are with College of Information Science and Engineering, Northeastern University.

Mailing address:

Northeastern University, College of Information Science and Engineering

P. O. Box 321, Shenyang, Liaoning, 110819, China

(C) 2015. This manuscript version is made available under the Elsevier user license http://www.elsevier.com/open-access/userlicense/1.0/ 


\title{
High Sensitivity Refractive Index Sensor Based on Splicing Points Tapered SMF-PCF-SMF Structure Mach-Zehnder Mode Interferometer
}

\author{
Qi Wang ${ }^{1,2, *}$, Lingxin Kong ${ }^{1}$, Yunli Dang ${ }^{1}$, Feng Xia ${ }^{1}$, Yongwei Zhang ${ }^{1}$, Yong Zhao ${ }^{1,2}$, Haifeng \\ $\mathrm{Hu}^{1}$, and $\mathrm{Jin}^{1}{ }^{1}$ \\ ${ }^{1}$ College of Information Science and Engineering, Northeastern University, Shenyang 110819, China
}

${ }^{2}$ State Key Laboratory of Synthetical Automation for Process Industries (Northeastern University), Shenyang 110819 , China

*Corresponding author Email: wangqi@ise.neu.edu.cn

\begin{abstract}
The paper proposed and studied a Mach-Zehnder mode interferometric refractive index sensor, which is based on splicing points tapered SMF-PCF-SMF (SMF, single-mode fiber; PCF, photonic crystal fiber) structure. For the reason that the effective refractive index of photonic crystal fiber cladding high-order modes near fiber core are more sensitive to surrounding refractive index changes, the refractive index measurement sensitivity of splicing points tapered SMF-PCF-SMF Mach-Zehnder mode interferometer can be enhanced further through tapering the splicing points. Relations between refractive index measurement sensitivity and photonic crystal fiber length and taper waist diameter are studied through numerical simulations and experiments. Simulation and experimental results show that sensitivity will be increased with the increase of photonic crystal fiber length and the decrease of taper waist diameter. In the refractive range of 1.3333-1.3737, splicing points tapered SMF-PCF-SMF Mach-Zehnder mode interferometer with PCF length of $4 \mathrm{~cm}$ and taper waist diameter of $60.4 \mu \mathrm{m}$ has refractive index measurement sensitivity of $260.8 \mathrm{~nm} / \mathrm{RIU}$, compared with sensitivity of $224.2 \mathrm{~nm} / \mathrm{RIU}$ of direct splicing SMF-PCF-SMF Mach-Zehnder mode interferometer with PCF length of $4 \mathrm{~cm}$, the sensitivity increased by $16.3 \%$. The research shows that the sensing structure is with good linearity and repeatability.

Keywords: Optical fiber sensor, Refractive index measurement, Mach-Zehnder interference, Photonic crystal fiber, High sensitivity, Splicing points taper
\end{abstract}

\section{Introduction}

Refractive index measurement has become one of the hot issues in the research of optical fiber sensing technology [1-2]. Liquid refractive index measurement based on optical method mainly has two methods: optical path measurement method and optical fiber measurement method [3]. Optical path measurement 
method is a common method, but optical path structure is complex and optical path is not stable. The structure of optical fiber measurement is simple, and it is the main method for refractive index measurement. The optical fiber sensor based on Mach-Zehnder interferometer has the advantages of fabrication simple and measuring convenient, which is a common method in the research of fiber optic interferometric sensors [4]. In 2008, Tian proposed a double tapered single-mode fiber Mach-Zehnder interferometer. It has the advantages of simple fabrication and low cost, but the refractive index measuring sensitivity is low. In refractive index range of 1.315 1.3618, the refractive index sensitivity is only $17.1 \mathrm{~nm} / \mathrm{RIU}$ [5]. In 2011, Geng proposed a new method of splicing points expansion method to make a Mach-Zehnder interference temperature sensor [6]. In recent years, photonic crystal fibers with periodic array of air holes have attracted attentions of researchers because of its high birefringence and nonlinear [7]. With the rapid development of PCF fabrication technology and rapid development of splicing technology, scholars have made many kinds of PCF interferometric fiber optic sensor [8-11]. In 2012, Wang proposed a method that splicing a short photonic crystal fiber between two sections of single-mode fiber to build a Mach-Zehnder interferometer through making air hole of photonic crystal fiber around the splice points collapse completely, and the resolution reaches $1.02 \times 10^{-4} \mathrm{RIU}$ in the refractive index range of $1.333 \sim 1.422$ [12]. In 2012, Liu made a tapered photonic crystal fiber Mach-Zehnder interferometer by chemical corrosion method, the sensitivity was 199nm/RIU[13]. Although this sensor has higher sensitivity, but the fabrication process of fiber corrosion is hard to control and the sensor has low mechanical strength. With the appearance of photonic crystal fiber, it has been widely used in fiber interferometer and sensor for its novel features and flexible structure. In 2015, Wu made a PCF taper-based Mach-Zehnder interferometer by tapering the PCF between two SMFs, the refractive index sensing sensitivity was 50.902nm/RIU [14]. The air holes of PCF were destroyed seriously in the process of preparation, which led to low sensitivity and high-loss.

The paper proposed and studied a kind of Mach-Zehnder mode interferometric refractive index sensor, which is based on splicing points tapered SMF-PCF-SMF (SMF, single-mode fiber; PCF, photonic crystal fiber) structure. This paper fabricated the sensor by direct splicing a short solid core photonic crystal fiber between two sections of single-mode fibers, and in the two splicing points taper regions, the higher order cladding modes can be stimulated and coupled between the SMF core and PCF cladding. For the reason that effective refractive index of photonic crystal fiber cladding high-order modes are more sensitive to surrounding refractive index changing, the refractive index measurement sensitivity of splicing points tapered SMF-PCF-SMF Mach-Zehnder mode interferometer can be further enhanced. The research shows that the sensing structure is with good linearity and repeatability. The refractive index sensitivity of splicing points tapered SMF-PCF-SMF structure $(260.8 \mathrm{~nm} / \mathrm{RIU})$ is 5 times higher than the sensitivity of PCF taper-based Mach-Zehnder interferometer (50.902nm/RIU) [14]. 


\section{Principle}

Fig. 1 shows the schematic of SMF-PCF-SMF structure Mach-Zehnder interferometer proposed in this paper. Fig. 1(a) is the schematic of direct fused SMF-PCF-SMF structure Mach-Zehnder interferometer, and Fig. 1(b) is the schematic of splicing point tapered SMF-PCF-SMF structure Mach-Zehnder interferometer. The Mach-Zehnder interferometer is based on the direct splicing type of SMF-PCF-SMF Mach-Zehnder interferometer structure (Fig. 1(a)), and then tapered the splice points to form a splice points tapered SMF-PCF-SMF structure Mach-Zehnder interferometer (Fig. 1(b)). In the experiments, the photonic crystal fiber and single-mode fiber are spliced together by a fiber fusion splicer, and then tapered the fused region using residual heat. When light passing through single-mode fiber into the first taper, for the reason that the fiber diameter becomes smaller, there will be more light coupling into photonic crystal fiber cladding, the light in fiber core and cladding will transmit at the same time, and then they reach the second taper, light in fiber cladding will be coupled back to fiber core and interference with light in fiber core. According to fiber evanescent field theory, the smaller the fiber diameter is, the stronger the fiber evanescent is. Since diameter of fiber taper region became smaller, so evanescent field at the fiber taper region will be enhanced. Therefore, light in the taper region will be more sensitive to the surrounding refractive index changing, so the refractive index sensing sensitivity of splice point tapered SMF-PCF-SMF structure Mach-Zehnder interferometer is higher than the traditional photonic crystal fiber Mach-Zehnder interferometer theoretically.

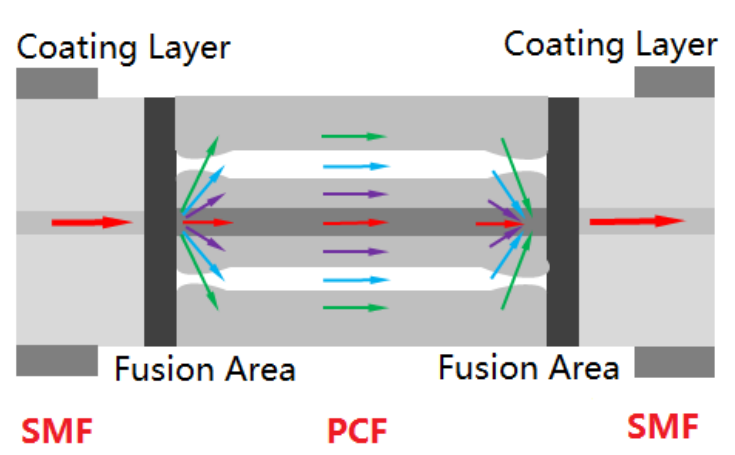

(a) Schematic of direct fused SMF-PCF-SMF structure MZI

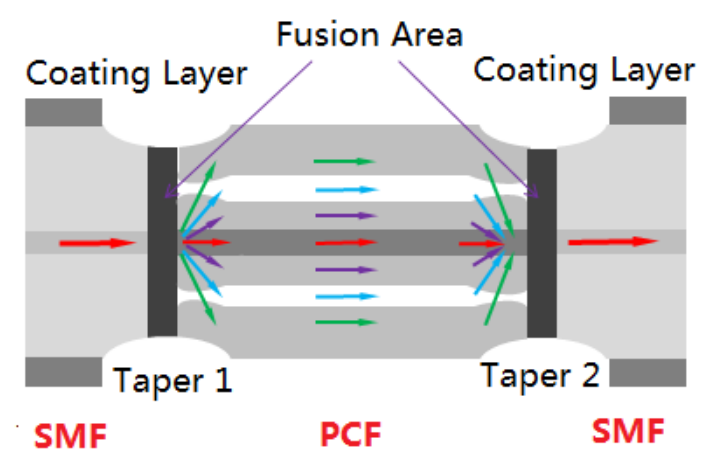

(b) Schematic of tapered SMF-PCF-SMF structure MZI

Fig. 1 Schematic of SMF-PCF-SMF structure MZI. SMF: Single mode fiber, PCF: Photonic crystal fiber

As it can be seen from Fig. 1, the splicing points tapered SMF-PCF-SMF structure forms a Mach-Zehnder interferometer. The tapered region in the fiber actually acts as couplers, and the first taper couples light in fiber core out into fiber cladding, and the second taper couples light in the fiber cladding into fiber core again to interfere with light in fiber core to form a Mach-Zehnder interferometer. The Mach-Zehnder interference can be expressed as Eq. (1). 


$$
I=I_{1}+I_{2}+2 \sqrt{I_{1} I_{2}} \cos \varphi
$$

Where $I_{1}$ and $I_{2}$ are the light intensity transmit in the fiber core and fiber cladding respectively, $\varphi$ is phase difference and it is shown in Eq. (2).

$$
\varphi=\frac{2 \pi \Delta n_{e f f} L}{\lambda}
$$

Where $L$ is optical route length of the interferometer, that is the length between the two splicing points taper, $\lambda$ is light wavelength, $\Delta n_{\text {eff }}$ is the difference between effective refractive index of fiber core and cladding, $\Delta n_{\text {eff }}=\mathrm{n}_{\text {eff }}^{\text {core }}-\mathrm{n}_{\text {eff }}^{\text {clading }}$. When difference between cladding mode and core mode equal to $(2 m+1) \pi$, interference between light in fiber core and cladding will result in destructive interference, that is to say, there will be an interference valley generated. The $m$-order interference valley can be concluded from Eq. (2) and it is shown in Eq. (3) [15-16].

$$
\lambda_{\mathrm{m}}=\frac{2 \Delta n_{e f f} L}{\lambda}
$$

Therefore, the free spectrum range (FSR) of the interference spectrum can be expressed as Eq. (4).

$$
\Lambda=\frac{\lambda^{2}}{\Delta \mathrm{n}_{e f f} L}
$$

Wavelength of the m-order interference valley will be changing along with the surrounding refractive index [17], and the variation is shown in Eq. (5).

$$
\Delta \lambda_{\mathrm{m}}=\frac{2\left(\Delta n_{\text {eff }}+\Delta n\right) L}{2 m+1}-\frac{2 \Delta n_{\text {eff }} L}{2 m+1}=\frac{2 \Delta n L}{2 m+1}
$$

Effective refractive index of photonic crystal fiber cladding is a function of surrounding refractive index, and effective refractive index of photonic crystal fiber core has nothing to do with surrounding refractive [18]. $\Delta n$ is the variation of effective refractive index of photonic crystal fiber cladding along with surrounding refractive index changing.

The cross section of the photonic crystal fiber used in this paper is shown in Fig. 2(a). The core and cladding of photonic crystal fiber is pure $\mathrm{SiO}_{2}$, refractive index of 1.457 , the cladding is composed of five layers of air holes, the fiber diameter is $125.2 \mu \mathrm{m}$, the diameter of the core is $8.89 \mu \mathrm{m}$ and air hole pitch is $7.67 \mu \mathrm{m}$, the air hole diameter is $4.88 \mu \mathrm{m}$. The single mode fiber is SMF-28, the core diameter is $8.3 \mu \mathrm{m}$, and the cladding diameter is $125 \mu \mathrm{m}$. The fusion splicer used in the experiments is s178 (Fitel, Inc.). Fig. 2(b) shows the single-mode fiber and photonic crystal fiber fusion splicing process. After completion of the fused taper, the effect of the fused taper was detected by using an ASE broadband light source $(1520 \mathrm{~nm} \sim 1570 \mathrm{~nm})$ 
and an optical spectrum analyzer (Yokogawa, AQ6370). In the fabrication process, manual splicing of PCF and SMF through adjusting splicer parameters. After completion of splicing, fiber tapering at the splicing point is carried out, and the waist diameter is tapered to $78 \mu \mathrm{m}$, and microscope image of PCF taper region is shown in Fig. 2(c).

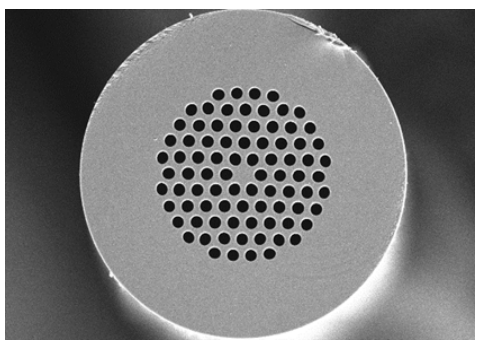

(a) Cross section of PCF

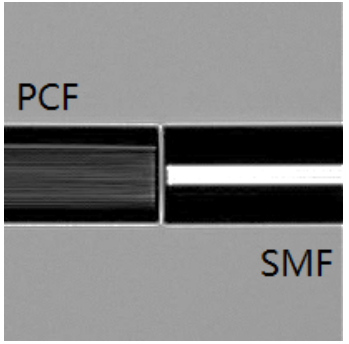

(b) Splicing process of SMF and PCF

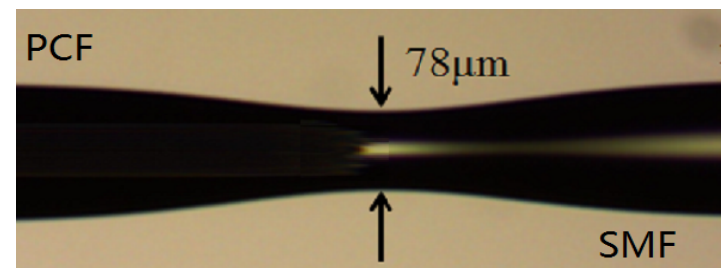

(c) Splicing point tapered SMF-PCF

Fig.2 Photonic crystal fiber and SMF-PCF splicing and taper

The Mach-Zehnder interferometer is formed by tapering the splicing points between photonic crystal fiber and single mode fiber. Compared with direct spliced SMF-PCF-SMF Mach-Zehnder interferometer, more high-order cladding modes can be stimulated in the splicing points tapered SMF-PCF-SMF Mach-Zehnder interferometer, and high-order modes of PCF cladding are more sensitive to external refractive index changing. Therefore, splicing points tapered SMF-PCF-SMF Mach-Zehnder interferometer will obtain greater sensitivity. The air hole diameter and hole spacing ratio of photonic crystal fiber used in the experiments is up to $66.7 \%$, therefore, compared to single-mode fiber Mach-Zehnder interferometric refractive index sensor, this splicing points tapered SMF-PCF-SMF Mach-Zehnder interferometer will get higher refractive index measurement sensitivity.

\section{Theoretical model and numerical simulation}

Fig. 3(a) is a schematic diagram of splicing points tapered single-mode fiber and photonic crystal fiber for numerical simulation. The model based on finite element simulation software and assume that the taper region collapse completely. Parameters of single-mode fiber used in the model are as follows: diameter and refractive index of SMF cladding is $125 \mu \mathrm{m}$ and 1.4613, diameter and refractive index of SMF core is $8.3 \mu \mathrm{m}$ and 1.4679 . The length of taper region is $60 \mu \mathrm{m}$, the taper waist diameter is $18 \mu \mathrm{m}$, and the input light wavelength is $1550 \mathrm{~nm}$. Fig. 3(b) shows the electric field energy distribution of splicing points tapered 
SMF-PCF-SMF structure. It can be seen from Fig. 3(b) that light transmitted in the SMF core will partially diffusion into PCF cladding and transmitted in the cladding and then recoupled back to SMF core and interference with SMF core fundamental mode.

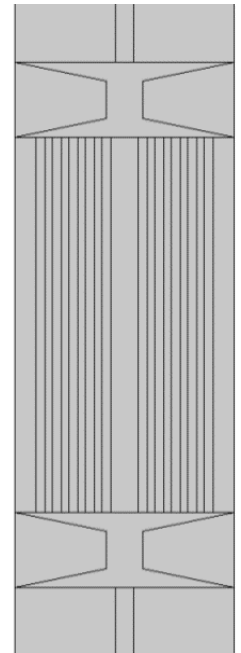

(a) Structure

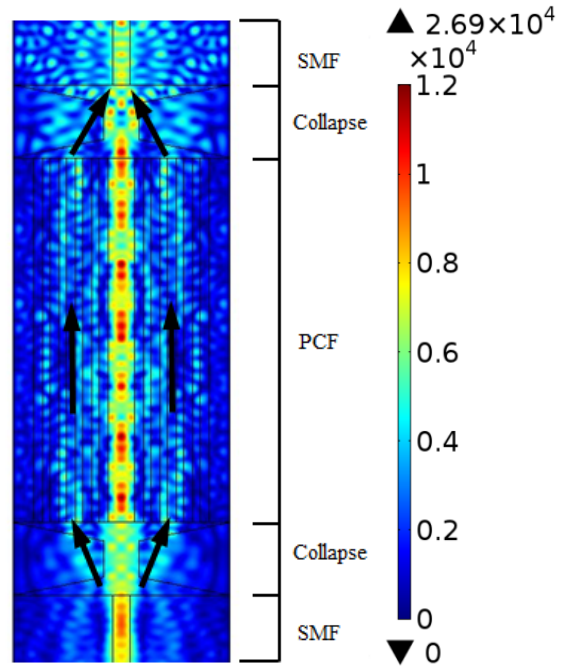

(b) Electric field energy distribution

Fig. 3 Numerical simulation of splicing points tapered SMF-PCF-SMF structure. $\lambda=1550 \mathrm{~nm}$, surface: Electronic field, $\mathrm{z}$ component $(\mathrm{V} / \mathrm{m})$. (The black arrow represent light transmitted direction, and the color from blue to red represents energy gradually more and more.)

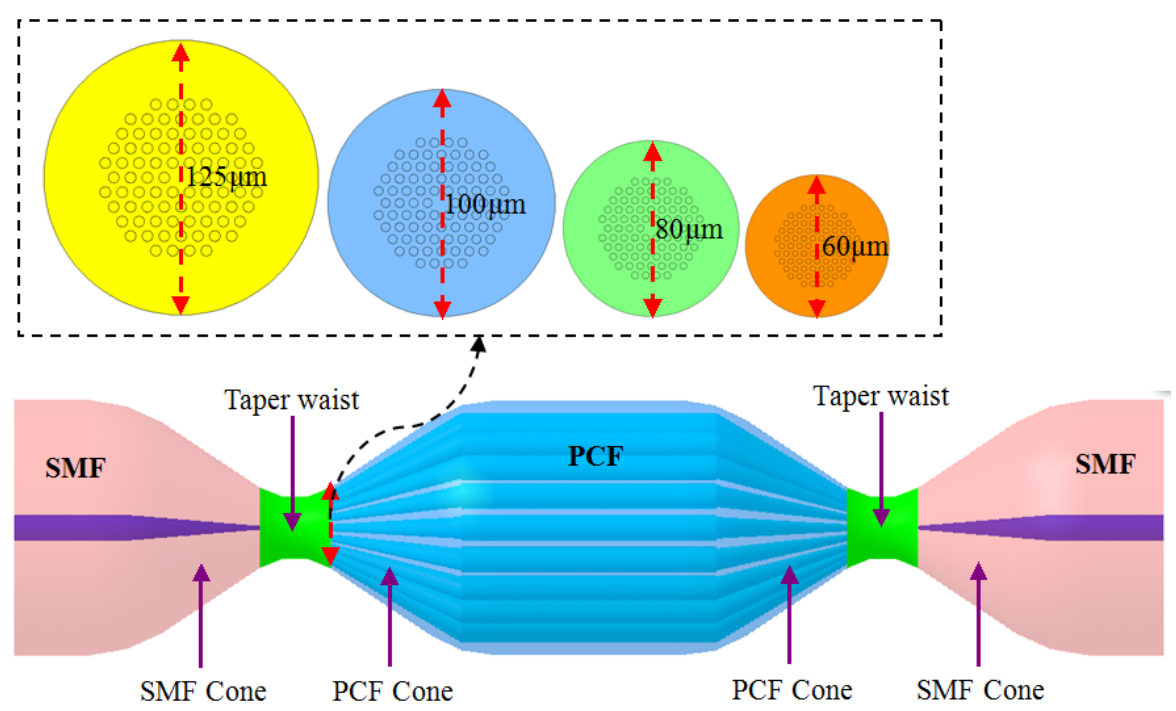

Fig. 4 Schematic diagram of splicing points tapered SMF-PCF-SMF Mach-Zehnder inerferometer

It is difficult to quantitatively analyze the influence of waist diameter on interference based on the above numerical simulations, so a splicing points tapered SMF-PCF-SMF Mach-Zehnder interferometer model which is more close to the actual situation was built as shown in Fig. 4. In the model, the splice points region was assumed completely collapse. As to single-mode fiber on one side of waist, a tapered cladding diameter and core diameter are considered as reduction in proportion. As to photonic crystal fiber on the other side of waist, the taper cladding diameter, core diameter and air hole diameter are also considered as reduction. The 
cascaded two taper in Fig. 4 are the same in structure. Fig. 4 shows the cross section of taper waist collapse area contact between photonic crystal fiber and single mode fiber at the photonic crystal fiber side. The diameter of the cross section is $60 \mu \mathrm{m}, 80 \mu \mathrm{m}, 100 \mu \mathrm{m}$, and $125 \mu \mathrm{m}$ respectively. Based on the finite element software COMSOL [19-20], the distribution and effective refractive index of fundamental mode and cladding mode field of photonic crystal fiber with different diameters are simulated, and the refractive index of the external solution is 1.35 .

Fig. 5 is the cladding mode (the model is equivalent to the seventh order cladding mode [21-22]) electric field energy distribution of the above four different photonic crystal fiber cross section diameter $(60 \mu \mathrm{m}, 80 \mu \mathrm{m}, 100 \mu \mathrm{m}$, and $125 \mu \mathrm{m})$. Because the diameter of a single-mode fiber is $8.3 \mu \mathrm{m}$, far less than $60 \mu \mathrm{m}$, so it is believed that higher-order modes excited at different diameter of the taper waist collapse area are approximately the same, we mainly consider the condition that same mode couple into the tapered photonic crystal fiber with different diameter propagating. Simulation results show that with taper waist diameter reducing from $125 \mu \mathrm{m}$ is to $60 \mu \mathrm{m}$, cladding mode energy gradually couple to the outside of photonic crystal fiber cross section. There will be more evanescent wave penetration into external solution, and it will cause cladding mode effective index gradually decreases.

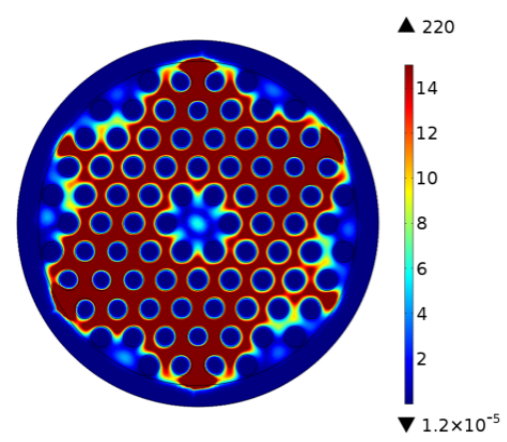

(a) Taper waist diameter $\phi=125 \mu \mathrm{m}$

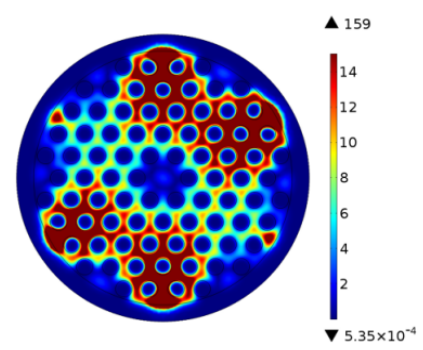

(c) Taper waist diameter $\phi=80 \mu \mathrm{m}$

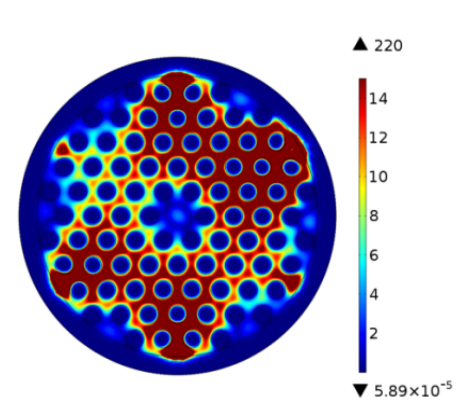

(b) Taper waist diameter $\phi=100 \mu \mathrm{m}$

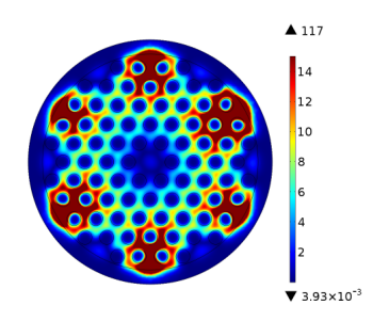

(d) Taper waist diameter $\phi=60 \mu \mathrm{m}$

Fig. 5 Cladding mode electric field energy distribution of different PCF taper waist diameter

Fig. 6(a) is numerical simulation results of surrounding solutions refractive index and effective refractive index difference between core mode and cladding mode $\left(\Delta n_{\text {eff }}=\mathrm{n}_{\text {eff }}^{\text {core }}-\mathrm{n}_{\text {eff }}^{\text {cladding }}\right)$ at taper waist diameter from $125 \mu \mathrm{m}$ to $20 \mu \mathrm{m}$. As it is shown in Fig. 6(a), when the taper waist diameter changing from 
$125 \mu \mathrm{m}$ to $40 \mu \mathrm{m}$, fundamental mode is the core mode and the $7^{\text {th }}$ order mode is equivalent to cladding mode (For the $7^{\text {th }}$ order mode excitation in good condition, approximately uniform distribution in the cladding [23]). When the taper waist diameter changing from $30 \mu \mathrm{m}$ to $20 \mu \mathrm{m}$, the first higher order mode has approximately uniform distribution in the cladding. At this time the first high order mode is equivalent to cladding mode. When the taper waist diameter below $10 \mu \mathrm{m}$, the basic mode cannot be confined in the photonic crystal fiber core.

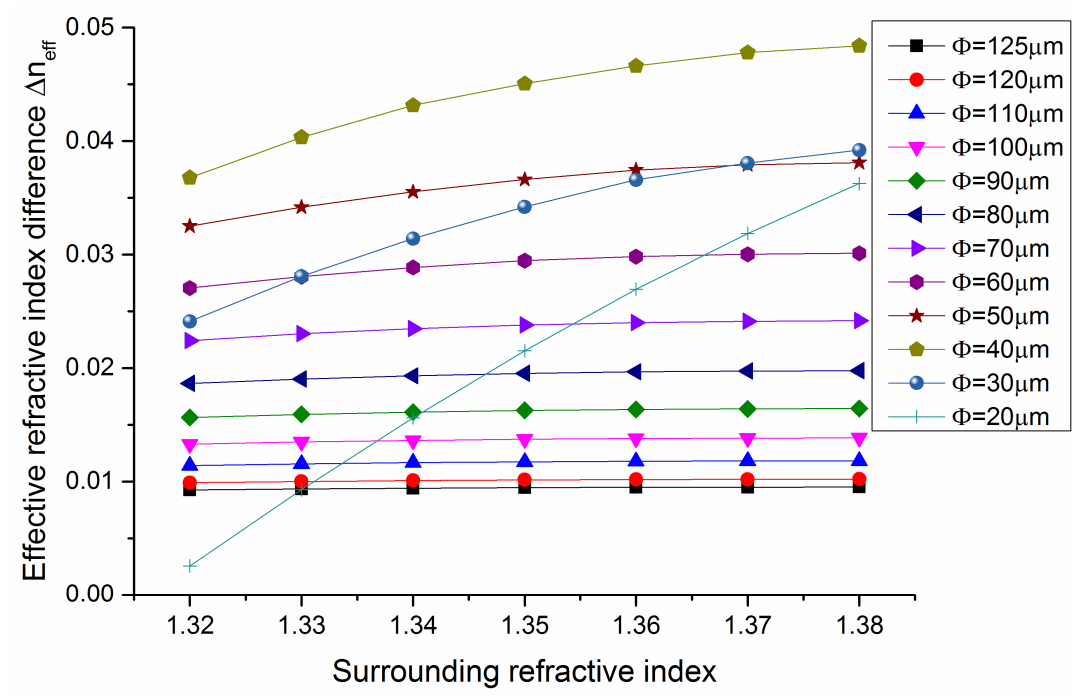

(a) Numerical simulation of relations between effective refractive index difference $\left(\Delta n_{\text {eff }}=\mathrm{n}_{\text {eff }}^{\text {core }}-\mathrm{n}_{\text {eff }}^{\text {clading }}\right)$ of different tapered waist diameter $(20 \mu \mathrm{m}-125 \mu \mathrm{m})$ PCF and surrounding refractive index.

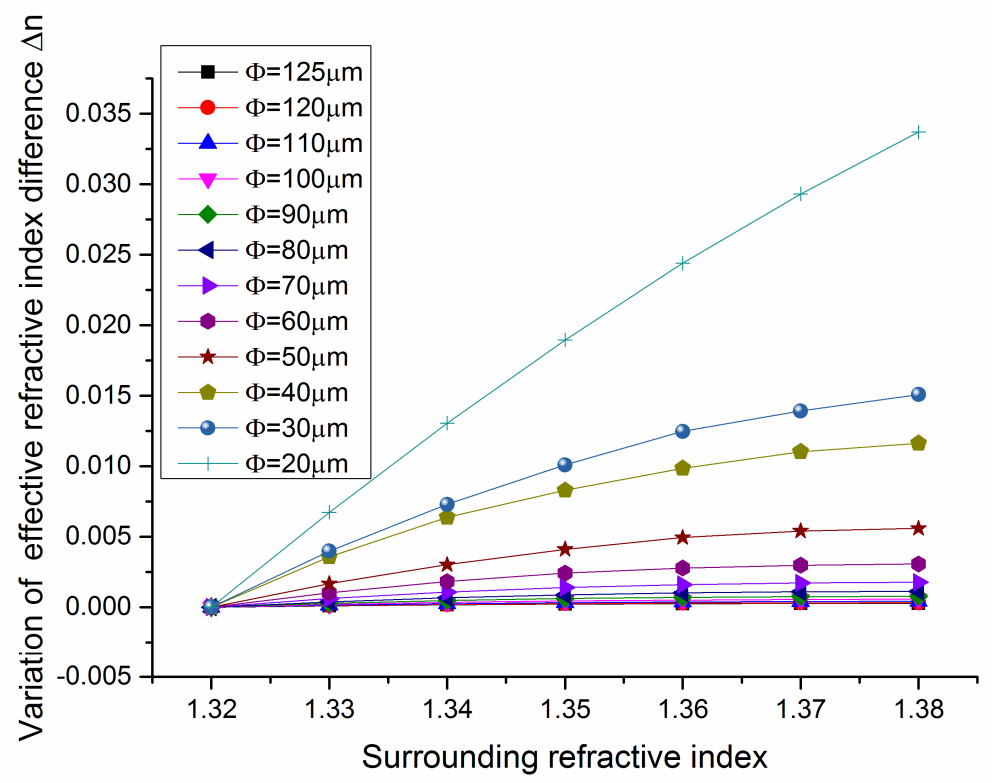

(b) Numerical simulation of relations between variation ( $\Delta n)$ of effective refractive index difference of different tapered waist diameter $(20 \mu \mathrm{m}-125 \mu \mathrm{m})$ PCF and surrounding refractive index.

Fig. 6 Relations between effective refractive index of tapered PCF and surrounding refractive index.

From Fig. 6(a) it can be seen, when the taper waist diameter in the range of $125 \mu \mathrm{m} \sim 20 \mu \mathrm{m}$, the 
surrounding refractive index changing from 1.32 to $1.38, \Delta n_{\text {eff }}$ has approximately linear relation with surrounding refractive index. When the surrounding refractive index is kept constant, $\Delta n_{\text {eff }}$ will increase with the taper waist diameter decreases from $125 \mu \mathrm{m}$ to $40 \mu \mathrm{m}$. The numerical simulation results indicate that decrease of taper waist diameter will excite higher order modes, and that will result in the increasing of $\Delta n_{\text {eff }}$ and further improve sensor sensitivity. When the taper waist diameter in the range of $30 \mu \mathrm{m} \sim 20 \mu \mathrm{m}$, as the decreasing of taper waist diameter, fitting slope of the curve in the figure gradually increased, which indicates that sensitivity of the sensor is improved with the decrease of taper diameter.

Fig. 6(b) is the relationship curve between surrounding solutions refractive index and variations of refractive index difference. It can be concluded from Fig. 6(b), with increase of surrounding refractive index, $\Delta n$ is increasing linearly, and the smaller the taper waist diameter is, the larger the curve fitting slope is. Therefore, based on Eq. (5), sensitivity of the splicing points tapered SMF-PCF-SMF Mach-Zehnder interferometer will increase with taper waist decrease.

It can be seen from effective refractive index simulation data of Fig. 6 that refractive index measurement sensitivity increase less when taper diameter lager than $50 \mu \mathrm{m}$, and when the taper waist diameter less than $50 \mu \mathrm{m}$, the sensitivity can be notably improved. This is mainly because effective refractive index of fundamental mode and cladding mode will both decrease with the decreases of taper waist diameter, and the increase ratio of $\Delta n_{\text {eff }}$ is reduced. Because of the complexity of the Mach-Zehnder mode interferometric, the sensitivity improvement need to be studied in the experiments.

\section{Experiments and discussion}

In experiments, the concentration of $\mathrm{NaCl}$ solution was $0 \%, 5 \%, 10 \%, 15 \%, 20 \%$ and $25 \%$ respectively, and the refractive index was measured. The relationship between concentration and refractive index is shown in Table 1. From Table 1, it can be seen that the $\mathrm{NaCl}$ solution concentration from $0 \%$ to $25 \%$ covers the range of common liquid refractive index.

Table 1 Relationship between $\mathrm{NaCl}$ solution concentration and refractive index

\begin{tabular}{ccccccc}
\hline $\mathrm{NaCl}$ solution concentration $(\%)$ & 0 & 5 & 10 & 15 & 20 & 25 \\
\hline refractive index & 1.3333 & 1.3411 & 1.3493 & 1.3574 & 1.3656 & 1.3737 \\
\hline
\end{tabular}

Experimental system apparatus is shown in Fig. 7. Experimental light source is amplified spontaneous emission (ASE) broadband light source, the wavelength range is $1520 \mathrm{~nm} \sim 1570 \mathrm{~nm}$. Optical spectrum analyzer (OSA, AQ6370) is produced by Yokogawa. Single-mode fiber is SMF-28. Photonic crystal fiber is an endlessly single mode photonic crystal fiber (NL-PCF-1, YOEC). Fig 7 (a) is schematic diagram of 
photonic crystal fiber Mach-Zehnder interferometer experimental system. In this paper, we first using the system studied direct spliced SMF-PCF-SMF Mach-Zehnder interferometer length (photonic crystal fiber length) of liquid measurement sensitivity effect. The photonic crystal fiber length were taken $2 \mathrm{~cm}, 3 \mathrm{~cm}$, and $4 \mathrm{~cm}$. Then, we studied the splicing points tapered SMF-PCF-SMF Mach-Zehnder interferometer in the same interference length (Length of photonic crystal fiber is $4 \mathrm{~cm}$ ) and different taper waist diameter (Diameter of taper waist $60.4 \mu \mathrm{m}, 78.8 \mu \mathrm{m}, 101.2 \mu \mathrm{m}$ respectively) of liquid measurement sensitivity effect through the system. The fiber sensing head fixed in the tested liquid pool connects to the broadband ASE light source and optical spectrum analyzer (OSA) in respectively through two single-mode fiber jumper. The ASE light source was used to provide wavelength range $1520 \mathrm{~nm}$ to $1570 \mathrm{~nm}$ input light and optical spectrum analyzer was used to display and analyze the output interference spectrum. Optical fiber was fixed stably on the measured liquid pool through fiber pressure foot, and the SMF-PCF-SMF Mach-Zehnder interferometer sensing head was kept straight and suspended in the liquid pool, so as to ensure the optical fiber sensing head can prevent small force interference outside and can fully contact with the liquid to be tested.

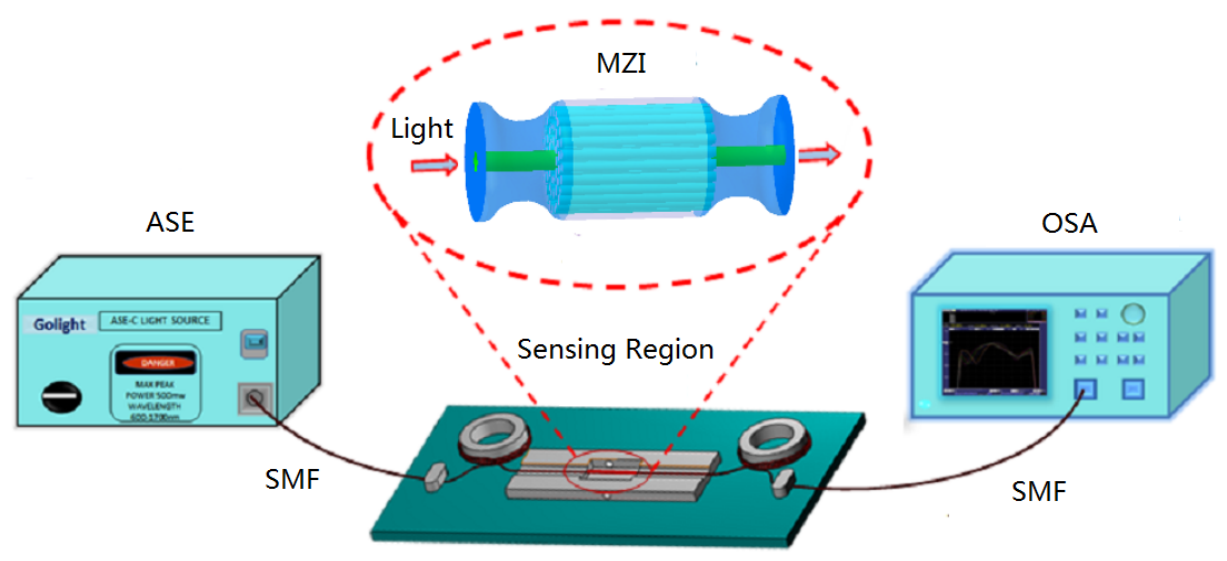

(a) Schematic diagram of splicing points tapered SMF-PCF-SMF Mach-Zehnder interferometer sensing system

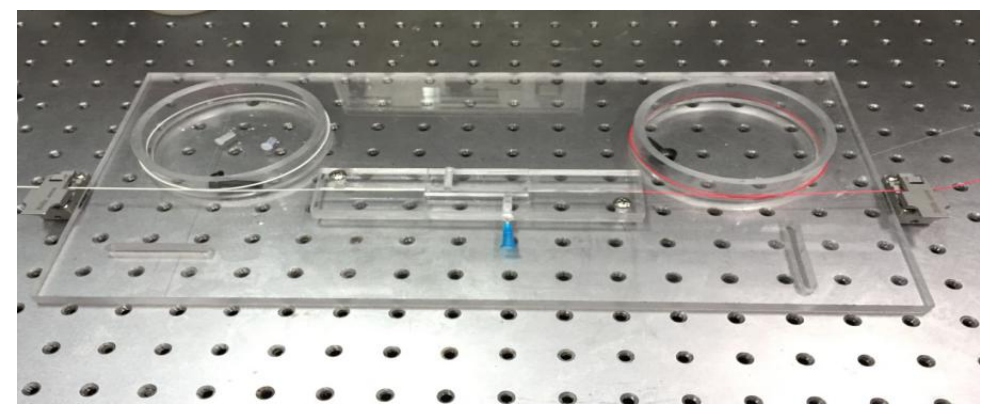

(b) Photo of sensing head

Fig. 7 Experimental setup of SMF-PCF-SMF Mach-Zehnder interferometer sensor

Photo of experimental system setup is shown in Fig. 7(b) and the specific experimental operations are as follows: (1) connecting the light source - optical fiber sensing head - optical spectrum analyzer; (2) the spectrum of fiber sensing head in the air is measured and saved as a reference spectrum; (3) The distilled 
water is injected into a liquid pool with a syringe, and then measured and saved the spectrum; (4) Liquid is removed from the liquid pool through the outlet, washing liquid pool with distilled water and alcohol alternately and repeatedly, drying liquid pool with compressed air, until the spectra the same as the reference spectra in the air. The interference spectra of $\mathrm{NaCl}$ solution with different refractive index were measured by the above steps. The average value of the three repeated measurements was performed to reduce the measurement error.

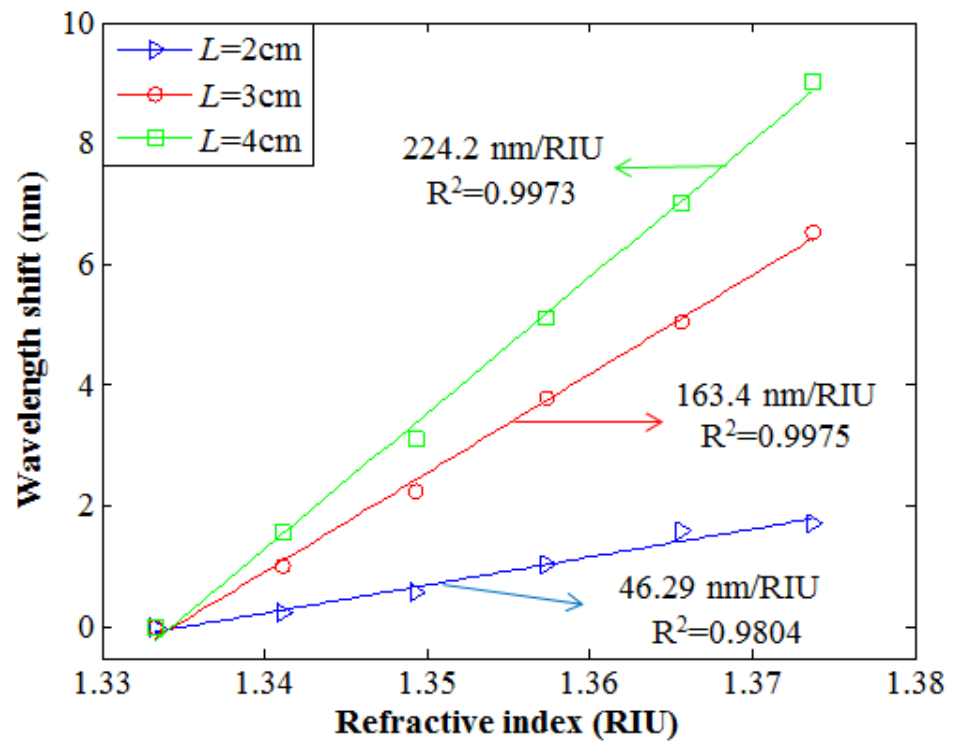

Fig. 8 Measuring sensitivity of direct splicing SMF-PCF-SMF Mach-Zehnder interferometer at different PCF length

Fig. 8 is a linear fitting curve of wavelength shift and refractive index at different photonic crystal fiber length, and the slope represents refractive index measurement sensitivity. It can be concluded from Fig. 8, when the photonic crystal fiber length is $\mathrm{L}=2 \mathrm{~cm}, 3 \mathrm{~cm}$, and $4 \mathrm{~cm}$, the refractive index measurement sensitivity is $46.29 \mathrm{~nm} / \mathrm{RIU}, 163.4 \mathrm{~nm} / \mathrm{RIU}$ and $224.2 \mathrm{~nm} / \mathrm{RIU}$ respectively, and the corresponding fitting degree $\left(\mathrm{R}^{2}\right)$ is $0.9804,0.9975$, and 0.9973 respectively. When $\mathrm{L}=4 \mathrm{~cm}$, the refractive index measurement sensitivity is $224.2 \mathrm{~nm} / \mathrm{RIU}$.
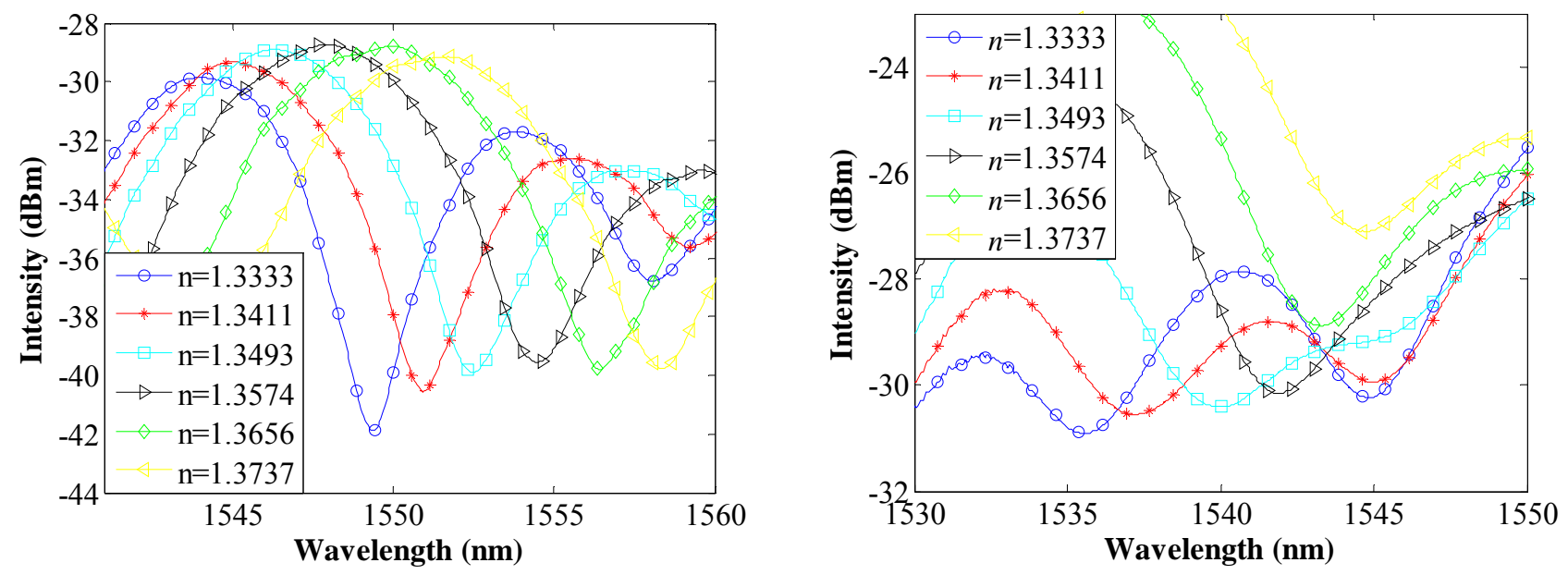
(a) Interference spectrum of direct splicing

SMF-PCF-SMF MZI.

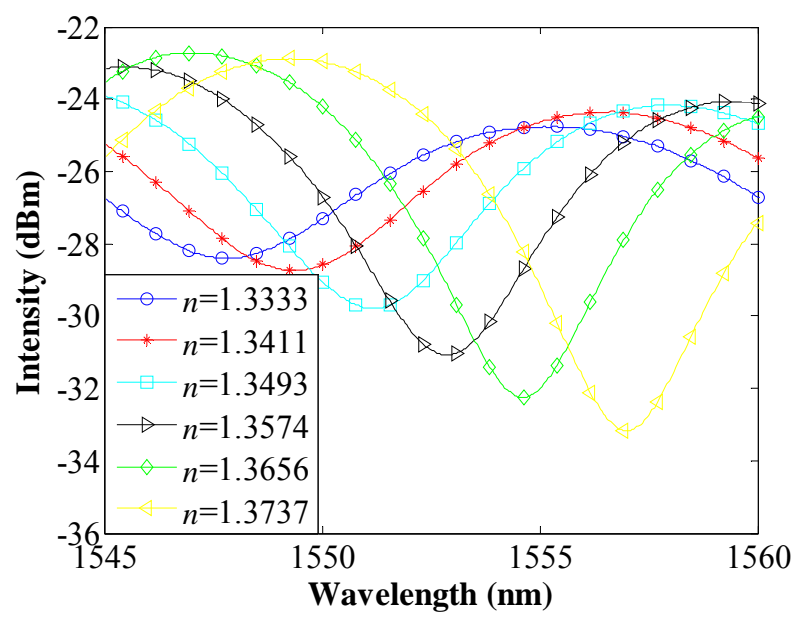

(c) Interference spectrum of splicing points tapered

SMF-PCF-SMF MZI at taper waist diameter of

$$
\phi=78.8 \mu \mathrm{m}
$$

(b) Interference spectrum of splicing points tapered

SMF-PCF-SMF MZI at taper waist diameter of $\phi=101.2 \mu \mathrm{m}$

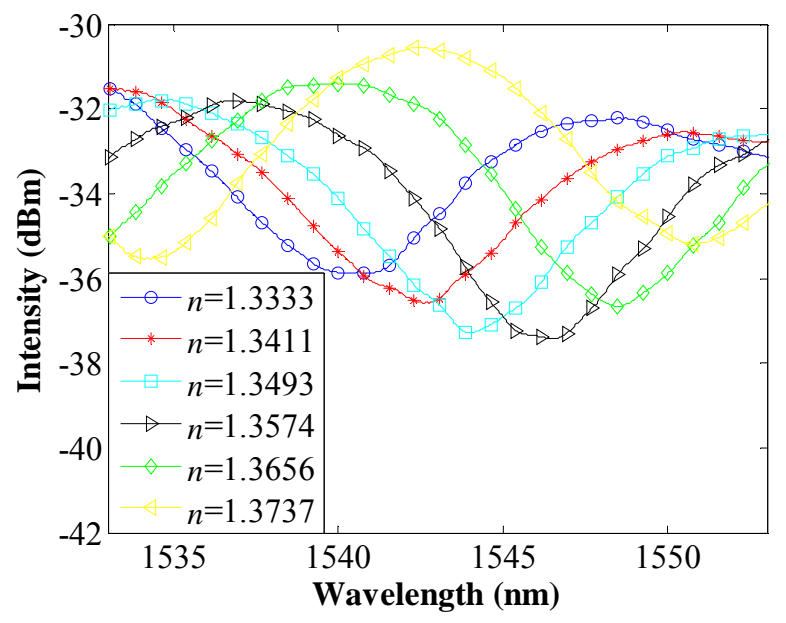

(d) Interference spectrum of splicing points tapered SMF-PCF-SMF MZI at taper waist diameter of

$$
\phi=60.4 \mu \mathrm{m}
$$

Fig. 9 Interference spectrum of direct splicing and splicing points tapered SMF-PCF-SMF Mach-Zehnder interferometer

Fig. 9 are interference spectra of direct splicing and splicing points tapered SMF-PCF-SMF Mach-Zehnder interferometer respectively. Based on the above studies, length of photonic crystal fiber of $4 \mathrm{~cm}$ is selected for the interferometer has the largest sensitivity. From Fig. 9 (a), (b), (c), (d) it can be seen that the refractive index measuring sensitivity of splicing points tapered SMF-PCF-SMF Mach-Zehnder interferometer is higher than direct splicing SMF-PCF-SMF Mach-Zehnder interferometer. The smaller the taper waist is, the larger the refractive index measurement sensitivity is. The sensitivity of refractive index measurement can reach $260.8 \mathrm{~nm} / \mathrm{RIU}$ at the taper waist diameter of $60.4 \mu \mathrm{m}$, which is increased by $16.3 \%$ from the sensitivity of $224.2 \mathrm{~nm} / \mathrm{RIU}$.

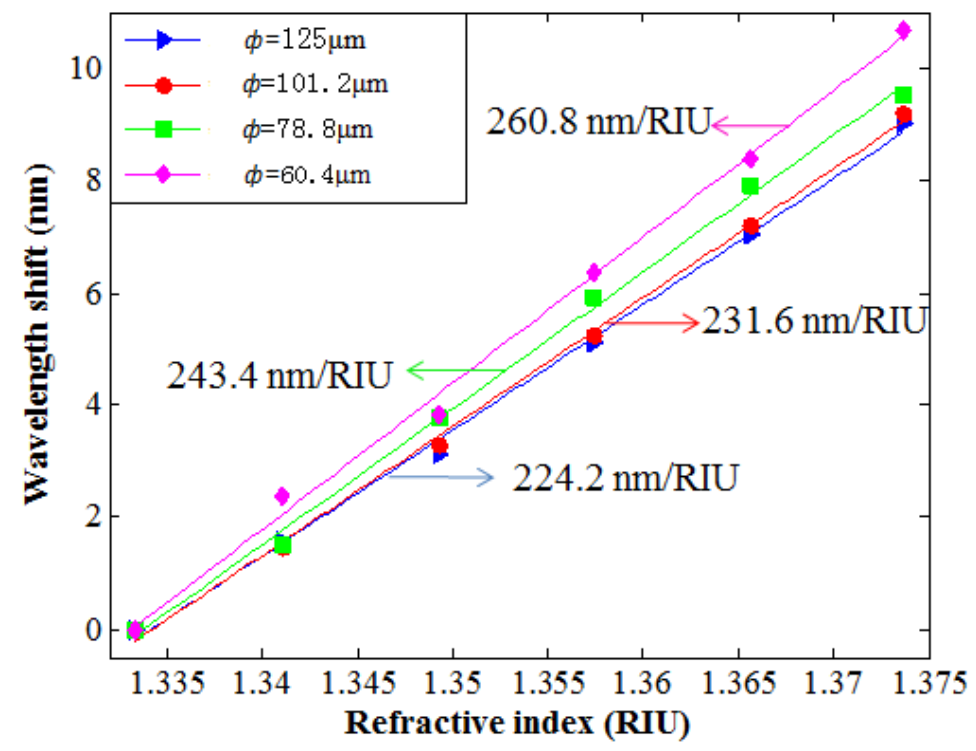

Fig. 10 Relations between wavelength shift and surrounding refractive index of direct splicing and splicing points tapered 
Fig. 10 is relations between wavelength shift and surrounding refractive index of direct splicing and splicing points tapered SMF-PCF-SMF Mach-Zehnder interferometer. From the experimental results of Fig. 10 , it can be seen that splicing points tapering can improve refractive index measuring sensitivity of SMF-PCF-SMF Mach-Zehnder interferometer. The reason is that, due to the first splice point tapering, the fiber diameter decreases will stimulate more high order modes which will infiltrate to external environment solution, and the contact area between cladding mode evanescent field and external solution increases, so surrounding refractive index changing will influence cladding mode transmission characteristics greater. According to refractive index definition, cladding mode effective refractive index can be reduced due to surrounding refractive index is smaller than cladding refractive index, and core mode effective refractive index will remain the same. Although with the core diameter becomes little, more fundamental mode energy will penetrate into the cladding, but due to cladding mode effective index has little difference with fundamental mode effective index, reduction amount fundamental mode effective index is lower than cladding mode. Based on Eq. (5), when surrounding refractive index is a constant, the taper waist diameter becoming small will result in $\Delta n_{\text {eff }}$ increasing, that is to say, it will result in wavelength shift increasing and sensitivity improvement, which also corresponding to the simulation results. The simulation and experimental results show that with taper waist diameter become smaller, the sensitivity can be further improved, but considering the fabrication process and mechanical strength, this paper haven't fabricated smaller diameter taper.

\section{Conclusion}

The paper proposed and studied a type of Mach-Zehnder mode interferometric refractive index sensor, which is based on splicing points tapered SMF-PCF-SMF (SMF, single-mode fiber; PCF, photonic crystal fiber) structure. This paper fabricated the sensor by direct splicing a short solid core photonic crystal fiber (PCF) between two sections of single-mode fibers, and in the two splicing points taper regions, the higher order cladding modes can be stimulated and coupled between the SMF core and PCF cladding. For the reason that effective refractive index of photonic crystal fiber cladding high-order modes are more sensitive to surrounding refractive index changes, the refractive index measurement sensitivity of splicing points tapered SMF-PCF-SMF Mach-Zehnder mode interferometer can be further enhanced. In this paper, relations between refractive index measurement sensitivity and photonic crystal fiber length and taper waist diameter are studied through numerical simulations and experiments. The simulation and experimental results show that sensitivity will increase with the increase of photonic crystal fiber length and the decrease of taper waist 
diameter. The experimental results show that interference spectrum central wavelength will emerge red shift as the surrounding refractive index increasing. In the refractive range of 1.3333-1.3737, direct splicing SMF-PCF-SMF Mach-Zehnder mode interferometer with PCF length of $4 \mathrm{~cm}$ has the sensitivity of 224.2nm/RIU, and splicing points tapered SMF-PCF-SMF Mach-Zehnder mode interferometer with PCF length of $4 \mathrm{~cm}$ and taper waist diameter of $60.4 \mu \mathrm{m}$ has refractive index sensitivity of $260.8 \mathrm{~nm} / \mathrm{RIU}$, that is to say the sensitivity increased by $16.3 \%$. The research shows that the sensing structure is with good linearity and repeatability.

\section{Acknowledgement}

This work was supported by the National Natural Science Foundation of China under Grant 61203206, the Fundamental Research Funds for the Central Universities under Grant N140405001, the Specialized Research Fund for the Doctoral Program of Higher Education of China under Grant 20120042120038, the National Science Foundation for Distinguished Young Scholars of China under Grant 61425003, the State Key Laboratory of Synthetical Automation for Process Industries under Grant 2013ZCX09, the Natural Science Foundation of Hebei Province under Grant F2014501137, the Natural Science Foundation of Liaoning Province under Grant 2013020010.

\section{Referecne}

1. Rajan G, Mathews S, Farrell G, et al. A liquid crystal coated tapered photonic crystal fiber interferometer[J]. Journal of Optics, 2011, 13(1):40-47.

2. Cooper PR. Refractive-index measurements of liquids used in conjunction with optical fibers[J]. Applied Optics, 1983, 22(19):3070-3072.

3. Singh S. Diffraction method measures refractive indices of liquids [J]. Physics Education, 2004, 39(3): 235.

4. Choi H Y, Kim M J, Lee B H. All-fiber Mach-Zehnder type interferometers formed in photonic crystal fiber[J]. Optics Express, 2007, 15(9):1-3.

5. Tian Z, Yam S H, Barnes J, et al. Refractive Index Sensing With Mach-Zehnder Interferometer Based on Concatenating Two Single-Mode Fiber Tapers[J]. IEEE Photonics Technology Letters, 2008, 20(8):626-628.

6. Geng Li, Tan, Deng, Yu. High Sensitivity Mach-Zehnder Interferometric Temperature Fiber Sensor Based on a Waist-Enlarged Fusion Bitaper[J]. IEEE Sensors Journal, 2011, 11(11): 2891-2894.

7. Samiul M. Habib, Selim M. Habib, M.I. Hasan, et al. A single mode ultra-flat high negative residual dispersion compensating photonic crystal fiber[J]. Optical Fiber Technology, 2014, 20: 328-332. 
8. J. Villatoro, V. P. Minkovich, V. Pruneri, G. Badenes. Simple all-microstructured-optical-fiber interferometer built via fusion splicing[J]. Optics Express, 2007, 15(4): 1491-1496.

9. H. Y. Choi, M. J. Kim, B. H. Lee. All-fiber Mach-Zehnder type interferometers formed in photonic crystal fiber[J]. Optics Express, 2007, 15(9): 5711-5720.

10. J. Villatoro, V. Finazzi, V. P. Minkovich, V. Pruneri, and G. Badenes. Temperature-insensitive photonic crystal fiber interferometer for absolute strain sensing[J]. Applied Physics Letters, 2007, 91(9) : 3.

11. J. Villatoro, M. P. Kreuzer, R. Jha, et al. Photonic crystal fiber interferometer for chemical vapor detection with high sensitivity[J]. Optics Express, 2009, 17(3): 1447-1453.

12. Wang J N, Tang J L. Photonic Crystal Fiber Mach-Zehnder Interferometer for Refractive Index Sensing[J]. Sensors, 2012, 12(3):2983-2995.

13. Liu Q, Wang Q. Refractive index sensor based on tapered PCF in-line interferometer[J]. Chinese Optics Letter, 2012, 10(9): 13-16.

14. Wu D, Zhao Y, Li J. PCF taper-based Mach-Zehnder interferometer for refractive index sensing in a PDMS detection cell[J]. Sensors \& Actuators B-Chemical, 2015, 213:1-4.

15. Villatoro J, Finazzi V, Minkovich V P, et al. Temperature-insensitive photonic crystal fiber interferometer for absolute strain sensing[J]. Applied Physics Letters, 2007, 91(9):091109-091109-3.

16. Niu L, Zhao C L, Gong H, et al. Curvature sensor based on two cascading abrupt-tapers modal interferometer in single mode fiber[J]. Optics Communications, 2014, 333:11-15.

17. H. C. Nguyen, B. T. Kuhlmey, E. C. Magi, et al. Tapered photonic crystal fibers: properties, characterization and applications[J]. Applied Physics B, 2005, 81(18):377-387.

18. R. Jha, J. Villatoro, G. Badenes, V. Pruneri. Refractometry based on a photonic crystal fiber interferometer[J]. Optics Letters, 2009, 34 (5):617-619.

19. Jiang Y Y, Liqing Y I, WEI YongTao amp. The finite element analysis of square-lattice multi-core photonic crystal fiber[J]. Scientia Sinica, 2011, 41(3):319-324.

20. Campos R E, Soares C J, Quagliatto P S, et al. In vitro study of fracture load and fracture pattern of ceramic crowns: a finite element and fractography analysis[J]. Journal of Prosthodontics Official Journal of the American College of Prosthodontists, 2011, 20(6):447-455.

21. T.A.Birks, J.C.Knight, P.St.J.Russell. Endlessly single-mode photonic crystal fiber[J]. Optics Letters, 1997, 22(13): 961-963.

22. Ren G. Study on Dispersion Properties of Photonic Crystal Fiber by Equivalent-Index Model[J]. Acta Optica Sinica, 2004, 24(3):319-323.

23. Wenwen Gu. Study on the transmission properties of optical waves in photonic crystal fibers[D]. Northwestern Polytechnical University. 2006. 


\section{Vitae}

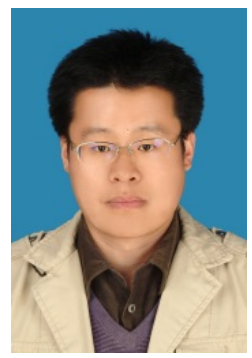

Qi Wang was born in Liaoning Province, China, in 1982. He received his Ph.D. degree in 2009 from the School of Physics and Optoelectronic Technology, Dalian University of Technology (DUT), Dalian, China. In 2013, he was awarded as the "Liaoning Bai-Qian-Wan Talents" by Liaoning Province. He is currently working as an associate professor in the College of Information Science and Engineering at Northeastern University, Liaoning Province, China. His research interests are new photonic devices, fiber-optic sensors, photonic crystal and photonic crystal fiber sensing technology, optoelectronic measurement technology and system, and their industrial applications. He has authored and co-authored more than 90 scientific papers, patents and conference presentations.

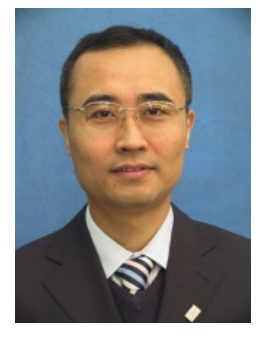

Yong Zhao received his M.A. and Ph.D. degrees, respectively, in precision instrument \& automatic measurement with laser and fiber-optic techniques from the Harbin Institute of Technology, China, in 1998 and 2001. He was awarded a first prize scholarship in 2000 by the China Instrument and Control Society and the Sintered Metal Corporation (SMC) scholarship in Japan. He was a scholarship in Japan. He was a post doctor in the Department of Electronic Engineering of Tsinghua University from 2001 to 2003, and then worked as an associate professor in the Department of Automation, Tsinghua University of China. In 2006, he was a visiting scholar of University of Illinois in Urbana and Champagne, USA. In 2008, he was awarded as the "New Century Excellent Talents in University” by the Ministry of Education of China. In 2009, he was awarded as the "Liaoning Bai-Qian-Wan Talents" by Liaoning Province. In 2011, he was awarded by the Royal Academy of Engineering as an academic researcher of City University London. In 2013, he was awarded as the "High Level Talents" by the Northeastern University. Now he is working in Northeastern University as a full professor. As a leader of his research group, his current research interests are the development of fiber-optic sensors and device, fiber Bragg grating sensors, novel sensor materials and principles, slow light and sensor technology, optical measurement technologies. He has authored and co-authored more than 200 scientific papers and conference presentations, 8 patents, and 5 books. He is a member in the Editorial Boards of the international journals of Sensor Letters, Instrumentation Science \& Technology, Journal of Sensor Technology, and Advances in Optical Technologies. 


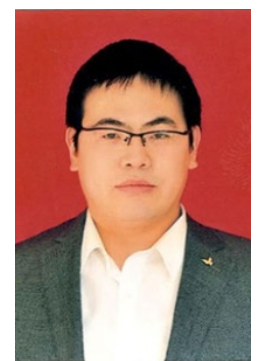

Jin Li was born in Yuncheng, Shan Xi Province, China in December 1983. He earned his bachelor degree in Electronic Science and Technology at Harbin Institute of Technology (HIT), China in 2007, Master's degree in Physical Electronics at HIT, China in 2009, Ph.D. degree in Physical Electronics at HIT, China in 2013. He is currently a Lecturer in the Department of Information Science and Engineering at Northeastern University, China since 2013. His research interests include micro- and nano-fiber sensor, surface plasmon, nanophotonics, nonlinear optics, laser-matter interaction and laser applications.

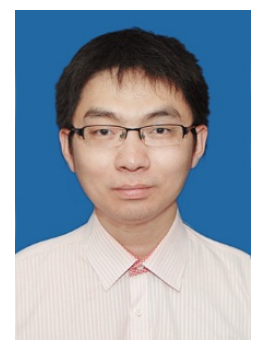

Haifeng Hu was born in Liaoning, China, in 1984. He received his Ph.D degrees in the Institute of Semiconductors, Chinese Academy of Sciences, China, in 2013. He is currently working in the College of Information and Engineering at Northeast University. His research interests are nano-optics, plasmonics, fiberoptic sensors and their applications in biosensing. He has authored and coauthored more than 30 scientific papers, 2 patents and 5 conference presentations. 